\title{
Energetics of edge oxidization of graphene nanoribbons
}

\author{
Airi Yasuma ${ }^{1 *}$, Ayaka Yamanaka ${ }^{2 \dagger}$, and Susumu Okada ${ }^{1 \ddagger}$ \\ ${ }^{1}$ Graduate School of Pure and Applied Sciences, University of Tsukuba, Tsukuba, Ibaraki 305-8571, Japan \\ Research Organization for Information Science and Technology (RIST), Minato, Tokyo 105-0013, Japan
}

\begin{abstract}
On the basis of the density functional theory, we studied the geometries and energetics of $\mathrm{O}$ atoms adsorbed on graphene edges for simulating the initial stage of the edge oxidization of graphene. Our calculations showed that oxygen atoms are preferentially adsorbed onto the graphene edges with the zigzag portion, resulting in a large adsorption energy of about $5 \mathrm{eV}$. On the other hand, the edges with armchair shape are rarely oxidized, or the oxidization causes substantial structural reconstructions, because of the stable covalent bond at the armchair edge with the triple bond nature. Furthermore, the energetics sensitively depends on the edge angles owing to the inhomogeneity of the charge density at the edge atomic sites.
\end{abstract}

\section{Introduction}

The exfoliation of graphite ${ }^{1,2}$ and the thermal annealing of $\mathrm{SiC}^{3,4}$ have now achieved the synthesis of the single-layered graphite, graphene, which provides us an ultimate two-dimensional electronic system with atom thickness. Much effort has been devoted to not only the elucidation of the fundamental physics of this new and famous carbon allotrope but also the exploration of the possibility of applications in future functional devices. ${ }^{5-8}$ Graphene possesses a pair of linear dispersion bands at the Fermi level and six corners of the Brillouin zone resulting in remarkable carrier mobility of 200,000 $\mathrm{cm}^{2} \mathrm{~V}^{-1} \mathrm{~s}^{-1}$, leading to the application of graphene in high-speed switching devices. ${ }^{9-11}$ The linear dispersion bands as well as the hexagonal covalent network allow us to tailor the electronic structures of graphene by imposing additional boundary conditions. The one-dimensional open boundary condition causes graphene strips of nanometer width (graphene nanoribbons), of which electronic structure is sensitive to the edge shapes and terminations. ${ }^{12-14}$ The graphene nanoribbons with hydrogenated armchair edges are semiconductors with a direct band gap at the $\Gamma$ point, which oscillates with triple periodicity of their width and asymptotically decreases with increasing width. In

\footnotetext{
${ }^{*}$ E-mail: ayasuma@comas.frsc.tsukuba.ac.jp

${ }^{\dagger}$ E-mail: yamanaka@rist.or.jp

${ }^{\ddagger}$ E-mail: sokada@comas.frsc.tsukuba.ac.jp
} 
contrast, the graphene nanoribbons with hydrogenated zigzag edges exhibit magnetic ordering around their edge atomic sites owing to half-filled flat-dispersion bands at the Fermi level around the zone boundary. In addition to the edge shapes, the electronic structure also substantially depends on the chemical functional groups attached to the edges. ${ }^{15-20}$ Hydroxylation of the graphene edges renders the peculiar delocalized state to the graphene edge. The state crosses the Fermi level and provides an electron channel in the vacuum spacing alongside the graphene edges with the free electron nature. ${ }^{18,19}$

The energetics of the graphene edges is less addressed than the electronic structures in terms of the edge variation. Several theoretical calculations have demonstrated that the energetics of graphene nanoribbons with clean and hydrogenated edges is sensitive to the edge shapes and nanoribbon width. ${ }^{21-27}$ Furthermore, the stability and geometric structures of graphene nanoribbons with zigzag and armchair edges have been theoretically studied. ${ }^{15,16,20}$ On the other hand, because of the large possible structures, the microscopic mechanism of the adsorption of atoms and functional groups onto graphene edges is still unclear. Thus, in this work, we aim to investigate the geometric structure and the energetics of the initial stage of the oxidization of graphene edges in terms of their edge shapes, using the density functional theory (DFT). Our calculations showed that the graphene edges consisting solely of the zigzag shape are preferentially oxidized with the large adsorption energy of about $5 \mathrm{eV}$, while the armchair edges are less preferable than the zigzag edges owing to the substantial structural reconstruction to reduce the large structural strain induced by the $\mathrm{C}-\mathrm{O}$ covalent bonds in some cases.

\section{Calculation methods and structural models}

All calculations were performed in the framework of $\mathrm{DFT}^{28,29}$ using the Simulation Tool for Atom TEchnology (STATE) package. ${ }^{30}$ To calculate the exchange-correlation energy among the interacting electrons, we used the generalized gradient approximation (GGA) with the functional forms of Perdew-Burke-Ernzerhof (PBE) ${ }^{31,32}$ Ultrasoft pseudopotentials generated using the Vanderbilt scheme were employed to describe the interaction between electrons and nuclei. ${ }^{33}$ The valence wave functions and deficit charge density were expanded in terms of the plane-wave basis set with cutoff energies of 25 and 225 Ry, respectively. Integration over the one-dimensional Brillouin zone was carried out using equidistant k-point sampling in which $4 \mathrm{k}$-points were taken along the ribbon direction, which give sufficient convergence in both electronic and geometric structures of graphene related materials. ${ }^{34,35}$ 
(a)

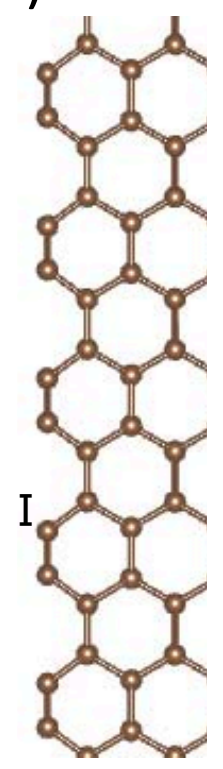

(b)

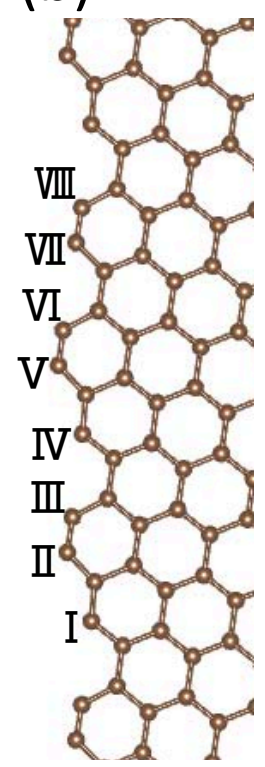

(c)

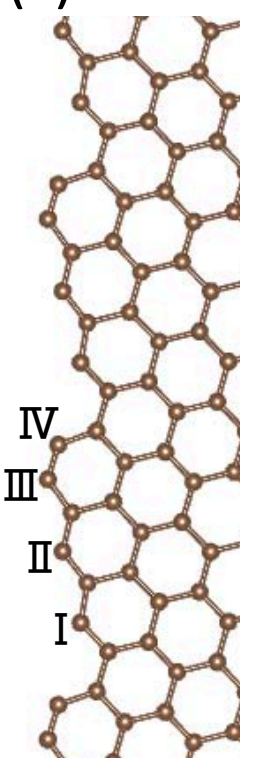

(d)

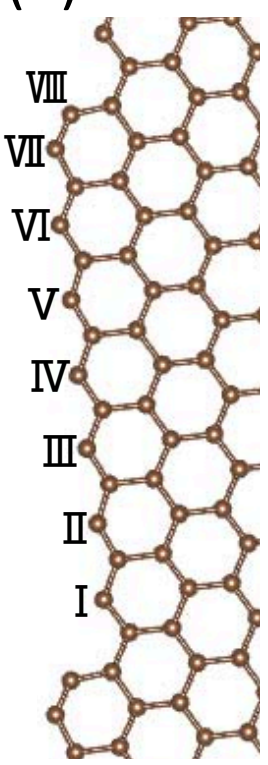

(e)

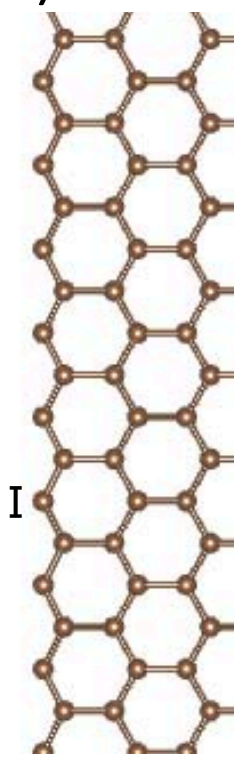

Fig. 1. Optimized geometries of graphene nanoribbons with (a) armchair edge $\left(\theta=0^{\circ}\right)$, chiral edges (b) $\theta=8^{\circ}$, (c) $\theta=16^{\circ}$, (d) $\theta=23^{\circ}$, and (e) zigzag edge $\left(\theta=30^{\circ}\right)$. The indexes show the independent edge atomic sites.

To simulate the geometric structure and energetics of edge oxidization of graphene with arbitrary edge shapes, we consider the graphene nanoribbons with clean edges, of which angles are 0 (armchair edge), 8, 16, 23, and 30 (zigzag edge) [Fig. 1]. To exclude the width dependence of the structures and energetics, each ribbon has a width of about $15 \AA$. As for the oxidization sites, we consider all independent edge atomic sites labeled as I - VIII in Fig. 1. For these edge atomic sites, we adsorbed a single $\mathrm{O}$ atom and performed structural optimization until the remaining force acting on each atom was less than 0.01 hartree/au under the fixed lattice constant along the ribbon direction, which corresponds to the length calculated using the experimental C-C bond length of bulk graphene $(1.42 \AA)$.

\section{Results and discussion}

Figure 2 shows the optimized structures of graphene nanoribbons, one of the edge atomic sites of which is terminated by an $\mathrm{O}$ atom with large, moderate, and small adsorption energies. The optimized structures under the $\mathrm{O}$ adsorption strongly depend on the edge shape and adsorption sites. The atomic configuration retains its initial structure when the $\mathrm{O}$ atom is attached to the zigzag atomic sites. In contrast, substantial structure relaxation occurs in the adsorbed structures where the $\mathrm{O}$ atom is attached to the $\mathrm{C}$ 


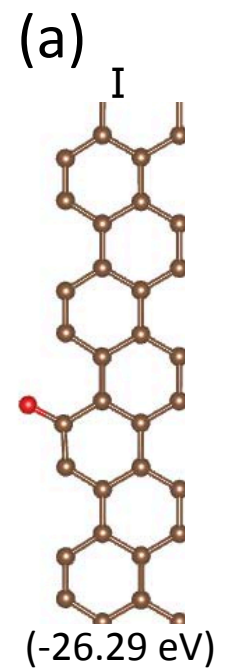

(b)

(c)
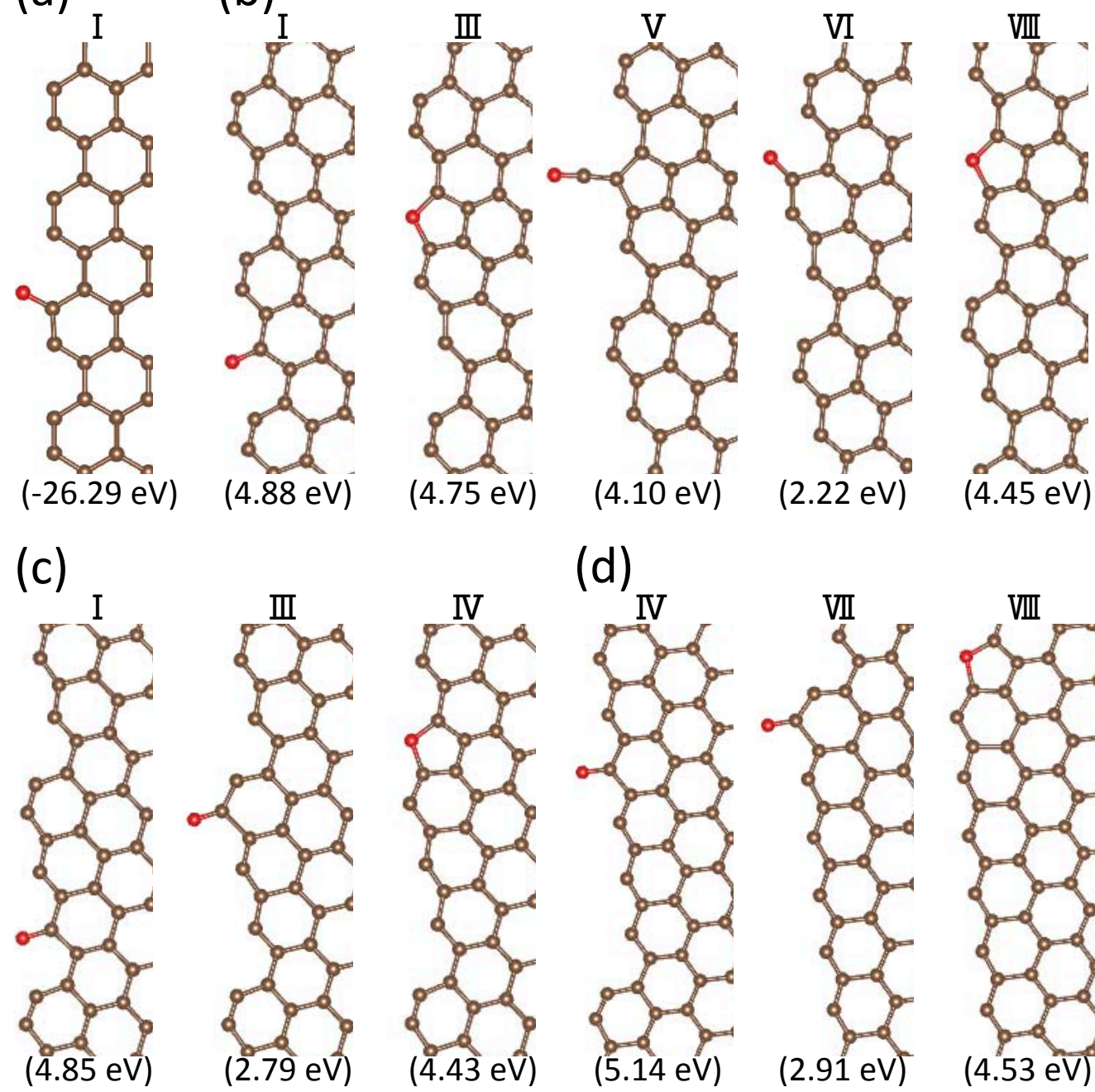

(4.45 eV)

(d)
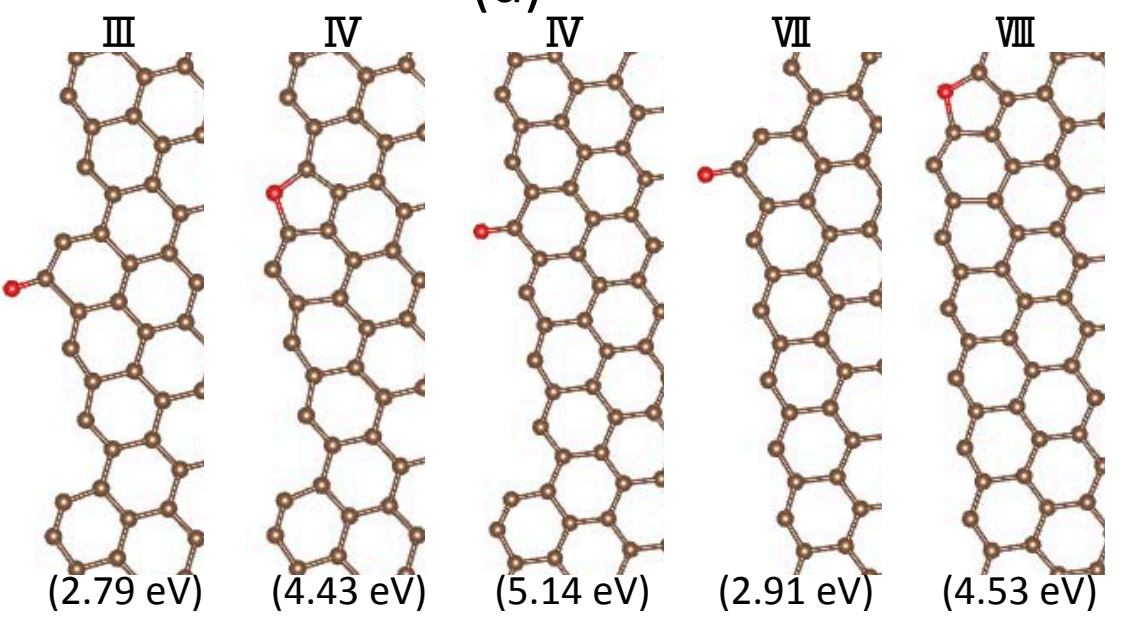

(e)

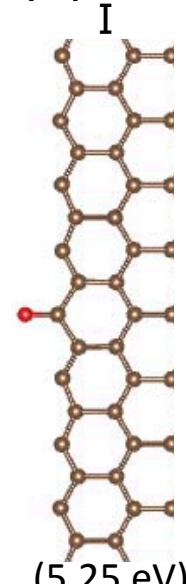

$(5.25 \mathrm{eV})$

Fig. 2. Optimized geometries of $\mathrm{O}$ adsorbed graphene nanoribbons with (a) the armchair edge $\left(\theta=0^{\circ}\right)$, chiral edges (b) $\theta=8^{\circ}$, (c) $\theta=16^{\circ}$, (d) $\theta=23^{\circ}$, and (e) zigzag edge $\left(\theta=30^{\circ}\right)$. The brown and red circles represent $\mathrm{C}$ and $\mathrm{O}$ atoms, respectively. The values in parentheses are the adsorption energies for the corresponding structures.

atoms situated at armchair sites[Fig. 2(a)]. Under the relaxed structures, the C-C bonds are longer by $0.15-0.18 \AA$ than those under the clean edges. Furthermore, when the O atom is situated at the cove region (atomic sites VIII, IV, and VIII in Figs. 1(b)- 1(d), respectively), the $\mathrm{O}$ atom bridges the cove and forms a pentagonal ring as its stable structure. Note that the CO chain together with the pentagonal ring has been formed when the $\mathrm{O}$ atom is attached to the atomic site $\mathrm{V}$ for the nanoribbon with the edge angle of $8^{\circ}$. In the structure $\mathrm{V}$ in Fig. 2(b), the edge CO may be easily dissociated under elevated temperature or other environmental conditions.

Table I summarizes the adsorption energy of the $\mathrm{O}$ atom to the edge atomic sites of 
Table I. Adsorption energy $\Delta E$ of an $\mathrm{O}$ atom to the clean edges of graphene nanoribbons.

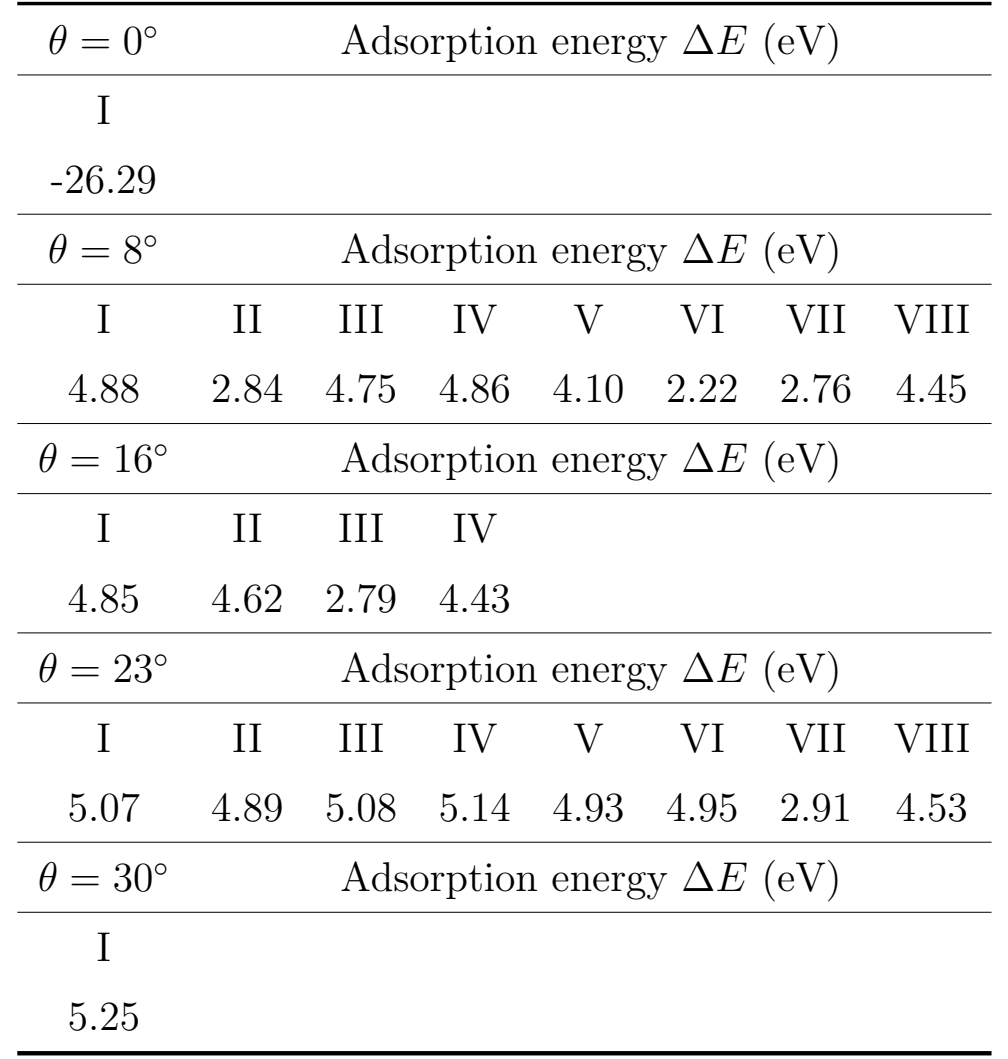

graphene nanoribbons with edge angles of $0,8,16,23$, and $30^{\circ}$. The adsorption energy $(\Delta E)$ is evaluated using the following formula:

$$
\Delta E=-\left(E_{G r / O}-E_{G r}-\mu_{O}\right)
$$

where $E_{G r / O}, E_{G r}$, and $\mu_{O}$ are the total energy of graphene nanoribbons with the O atom, that of graphene nanoribbons with clean edges, and the chemical potential of the $\mathrm{O}$ atom evaluated with an isolated $\mathrm{O}_{2}$ molecule, respectively. The $\mathrm{O}$ adsorption is exothermic for all atomic sites and edge angles except the clean armchair edge (the edge angle of $0^{\circ}$ ). According to the $\mathrm{O}$ adsorbed structures, the adsorption energy also depends on the adsorbed atomic site and edge angles. For the atomic sites at the zigzag region, the adsorption energies range from 4.62 to $5.25 \mathrm{eV}$, indicating that $\mathrm{C}$ atoms situated at the zigzag edge are preferentially oxidized under $\mathrm{O}$ exposure, because the edge atomic site with the zigzag shape is energetically unstable owing to the unsaturated covalent bond. In contrast, the adsorption energies for the edge sites with armchair shapes range from 2.22 to $2.91 \mathrm{eV}$, which is smaller than those for the zigzag edges. Therefore, the armchair edges are less reactive than the zigzag edges, because the armchair edge is 


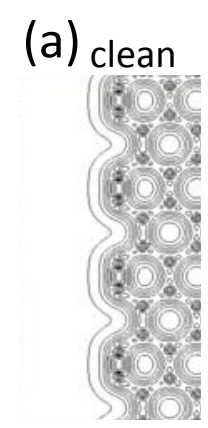

(c)

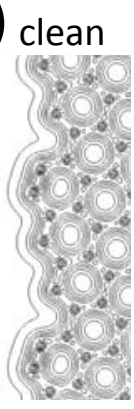

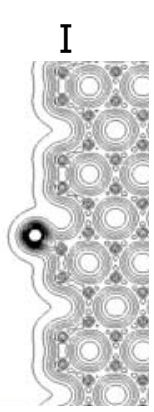

(b)

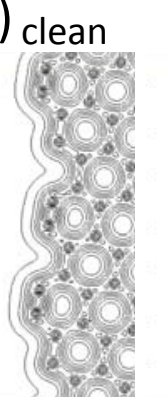

I

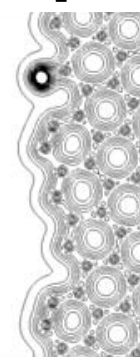

III

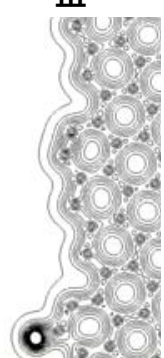

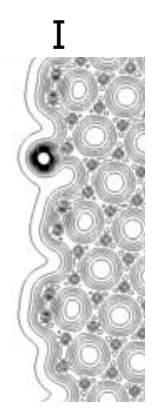

IV

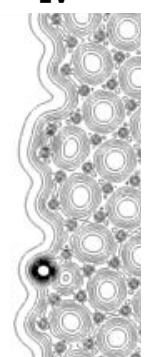

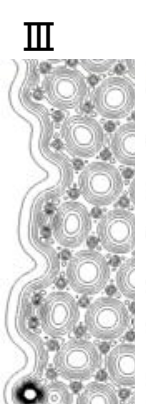

(d)

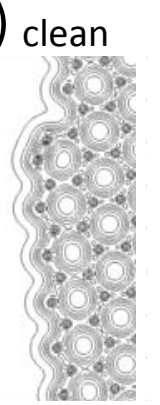

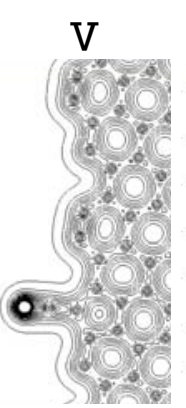
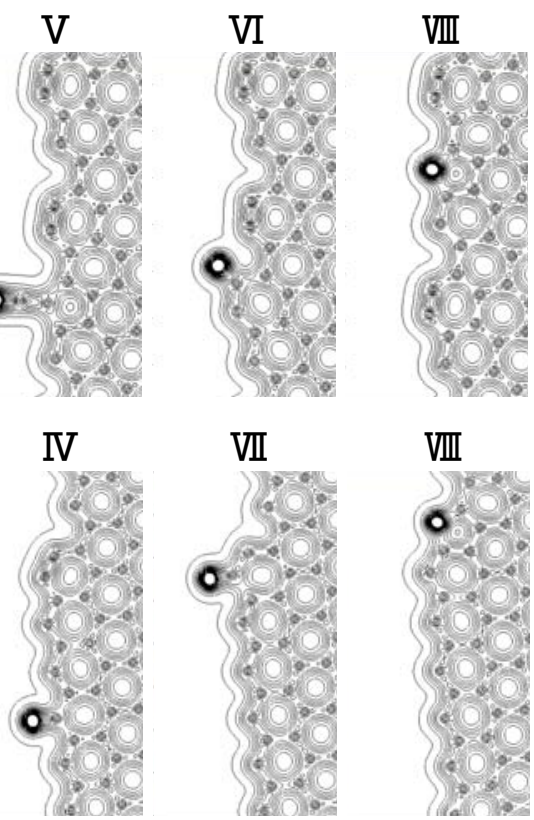

(e) clean

\section{I}

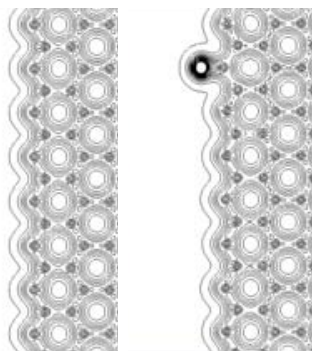

Fig. 3. Contour plots of the valence charge density of clean and edge oxidized graphene nanoribbons with edge angles of (a) 0, (b) 8, (c) 16, (d) 23, and (e) $30^{\circ}$. The index in each panel corresponds to the structures shown in Fig. 2. The adjacent contours denote the electron density of $0.05 \mathrm{e} / \mathrm{au}$.

energetically stable by forming a triple bond for reducing the dangling bond nature. For the cases of the oxidized edges with a pentagonal ring, the adsorption energy is relatively large, indicating that the cove region of the edges is also a reactive site for oxygen adsorption. Note that the formation of a triangle by $\mathrm{O}$ and edge $\mathrm{C}$ atoms results in a small adsorption energy (about $1.5 \mathrm{eV}$ ) because the resultant structure is energetically unstable.

To give a theoretical insight into the energetics of the $\mathrm{O}$ adsorption on graphene edges, we investigate the valence charge density of graphene nanoribbons with edge oxidization. Figure 3 shows the contour plots of the charge density of graphene nanoribbons before and after the edge oxidization. The oxidization at the zigzag edges does not affect the charge density of $\mathrm{C}-\mathrm{C}$ covalent bonds, reflecting the fact that the structural recon- 
struction upon oxidization is negligible for the zigzag edges. In contrast, the oxidization at the armchair edges causes the charge density modulation around the oxidized edge atomic sites. Because of the elongation of armchair $\mathrm{C}-\mathrm{C}$ bonds at the edge, the charge density on this bond substantially decreases upon $\mathrm{O}$ adsorption. Thus, the small adsorption energy near the armchair edges is ascribed to the substantial modulation of the charge density of armchair covalent bonds, which causes the dangling bond nature at the edge $\mathrm{C}$ atoms adjacent to the $\mathrm{C}$ atom attaching to the $\mathrm{O}$ atom. For the cases of the edge reconstruction forming a pentagonal ring, we find a finite charge density between the atoms forming pentagonal rings at the edges. Thus, the covalent bonds are formed between $\mathrm{C}$ and $\mathrm{O}$ atoms in the pentagonal ring.

Finally, we demonstrate the electronic energy band of edge oxidized graphene nanoribbons (Fig. 4). Because the pristine nanoribbons with clean edges possess unsaturated bonds at their edges except the armchair nanoribbon, the Fermi level is pinned at the dangling bond states associated with the edge $\mathrm{C}$ atoms. Even though the oxidization causes an increase or decrease in the electron states associated with dangling bonds, the Fermi level is still pinned at the dangling bond states. However, the oxidization substantially modulates the $\pi$ electronic states near the Fermi level by modulating the electrostatic potential of $\mathrm{C}$ atoms connected to the $\mathrm{O}$ atom. Some of the energy bands associated with the $\pi$ electron states of nanoribbons near the Fermi level open the finite gap of about $0.5 \mathrm{eV}$. In addition, the electronic structure weakly depends on the adsorption site of the $\mathrm{O}$ atom. Note that the number of dangling bond states and the edge $\mathrm{C}-\mathrm{O}$ structure cause the band structure modulation near the Fermi level of about a few hundreds meV.

\section{Conclusions}

We investigated the geometric structure and energetics of the initial stage of the oxidization of graphene edges in terms of their edge shapes, on the basis of the DFT. Our calculations showed that the graphene edges consisting solely of the zigzag shape are preferentially oxidized with the large adsorption energy of about $5 \mathrm{eV}$, while the armchair edges are less preferable than the zigzag edges, because of the substantial structural reconstruction upon $\mathrm{O}$ adsorption, which causes the dangling bond states at the edge $\mathrm{C}$ atoms adjacent to the oxidized $\mathrm{C}$ atom. In addition, $\mathrm{O}$ adsorption to the cove region of the armchair edge forms a pentagonal ring by bridging the cove. According to the structural reconstruction, the oxidized nanoribbons comprising the pentagonal ring 

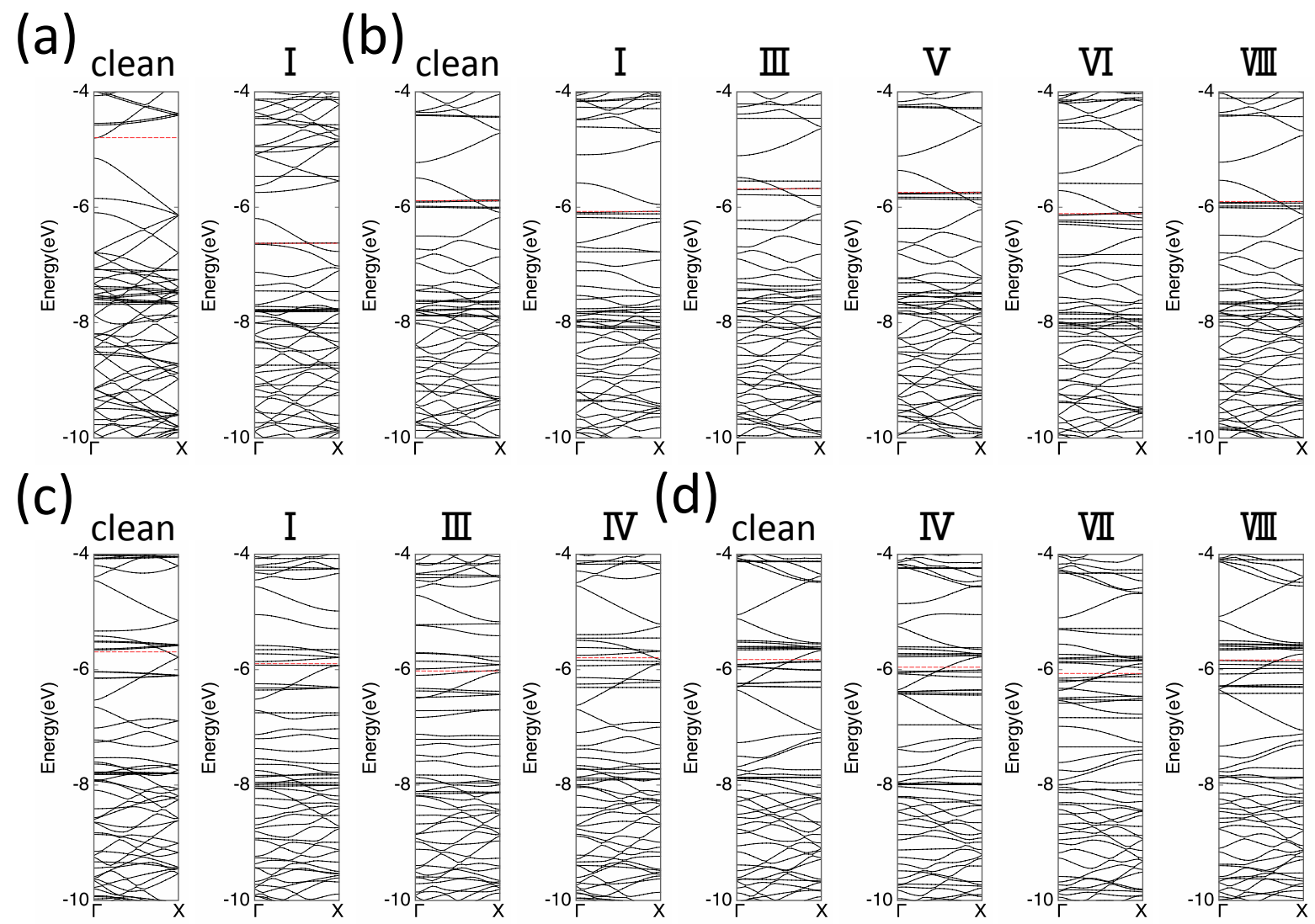

(d)
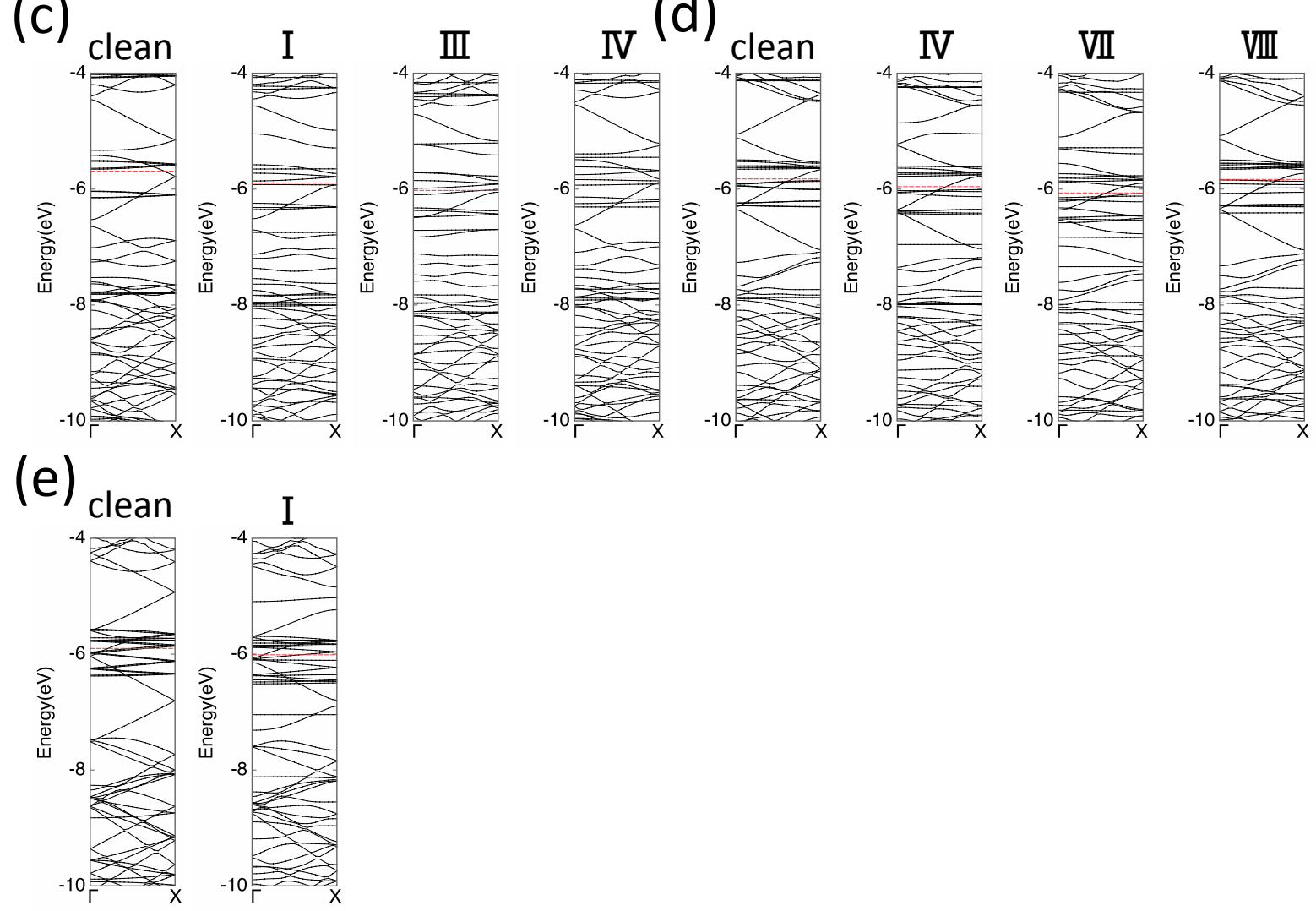

Fig. 4. Energy band structures of clean and edge oxidized graphene nanoribbons with edge angles of (a) 0, (b) 8, (c) 16, (d) 23, and (e) 30 . The index in each panel corresponds to the structures shown in Fig. 2. The horizontal dotted lines denote the Fermi level.

at the edge show remarkable energetic stability, and the energy gain upon $\mathrm{O}$ adsorption is the same as that with the ground-state conformation. In contrast, the oxygen adsorption onto the bridging site of the edge $\mathrm{C}-\mathrm{C}$ bond is the least stable oxidized structure. The electronic structures of the graphene nanoribbons are substantially modulated by the edge oxidization, while it is insensitive to the oxidized structures. 


\section{Acknowledgements}

This work was supported by JST-CREST Grant Numbers JPMJCR1532 and JPMJCR1715 from the Japan Science and Technology Agency, JSPS KAKENHI Grant Numbers JP17H01069, JP16H00898, and JP16H06331 from the Japan Society for the Promotion of Science, and the Joint Research Program on Zero-Emission Energy Research, Institute of Advanced Energy, Kyoto University. Part of the calculations was performed on an NEC SX-Ace at the Cybermedia Center at Osaka University and on an SGI ICE XA/UV at the Institute of Solid State Physics, The University of Tokyo. 


\section{References}

1) K. S. Novoselov, A. K. Geim, S. V. Morozov, D. Jiang, Y. Zhang, S. V. Dubonos, I. V. Grigorieva, and A. A. Firsov, Science 306, 666 (2004).

2) A. K. Geim and K. S. Novoselov, Nat. Mater. 6, 183 (2007).

3) I. Forbeaux, J.-M. Themlin, and J.-M. Debever, Phys. Rev. B 58, 16396 (1998).

4) C. Berger, Z. Song, X. Li, X. Wu, N. Brown, C. Naud, D. Mayou, T. Li, J. Hass, A. N. Marchenkov, E. H. Conrad, P. N. First, and W. A. de Heer, Science 312, 1191 (2006).

5) K. S. Novoselov, A. K. Geim, S. V. Morozov, D. Jiang, M. I. Katsnelson, I. V. Grigorieva, S. V. Dubonos, and A. A. Firsov, Nature 438, 197 (2005).

6) Y. Zhang, Y.-W. Tan, H. L. Stormer, and P. Kim, Nature 438, 201 (2005).

7) J. B. Oostinga, H. B. Heersche, X. Liu, A. F. Morpurgo, and L. M. K. Vandersypen, Nat. Mater. 7, 151 (2007).

8) Y. Zhang, T.-T. Tang, C. Girit, Z. Hao, M. C. Martin, A. Zettl, M. F. Crommie, Y. R. Shen, and F. Wang, Nature 459, 820 (2009).

9) M. Posternak, A. Baldereschi, A. J. Freeman, E. Wimmer, and M. Weinert, Phys. Rev. Lett. 50, 761 (1983).

10) M. Posternak, A. Baldereschi, A. J. Freeman, and E. Wimmer, Phys. Rev. Lett. 52, 863 (1984).

11) G. W. Semenoff, Phys. Rev. Lett. 53, 2449 (1984).

12) M. Fujita, K. Wakabayashi, K. Nakada, and K. Kusakabe, J. Phys. Soc. Jpn. 65, 1920 (1996).

13) K. Nakada, M. Fujita, G. Dresselhaus, and M. S. Dresselhaus, Phys. Rev. B 54, 17954 (1996).

14) Y. Miyamoto, K. Nakada, and M. Fujita, Phys. Rev. B 59, 9858 (1999).

15) D. Gunlycke, J. Li, J. W. Mintmire, and C. T. White, Appl. Phys. Lett. 91, 112108 (2007).

16) D. G. Kvashnin, P. B. Sorokin, J. W. Brüning, and L. A. Chernozatonskii, Appl. Phys. Lett. 102, 183112 (2013).

17) H. Jippo and M.Ohfuchi, J. Appl. Phys. 113, 183715 (2013).

18) R. Taira, A. Yamanaka, and S. Okada, Appl. Phys. Express 9, 115102 (2016).

19) R. Taira, A. Yamanaka, and S. Okada, Jpn. J. Appl. Phys. 56, 085103 (2017).

20) E. Carbonell-Sanromà, J. Hieulle, M. Vilas-Varela, P. Brandimarte, M. Iraola, A. 
Barragán, J. Li, M. Abadia, M. Corso, D. Sánchez-Portal, D. Peña, and J. I. Pascual, ACS Nano 11, 7355 (2017).

21) V. Barone, O. Hod, and G. E. Scuseria, Nano Lett. 6, 2748 (2006).

22) S. Okada, Phys. Rev. B 77, 041408 (2008).

23) B. Huang, M. Liu, N. Su, J. Wu, W. Duan, B. L. Gu, and F. Liu, Phys. Rev. Lett. 102, 166404 (2009).

24) P. Koskinen, S. Malola, and H. Häkkinen, Phys. Rev. Lett. 101, 115502 (2008).

25) C. K. Gan and D. J. Srolovitz, Phys. Rev. B 81, 125445 (2010).

26) S. Suda and A. Oshiyama, J. Phys. Soc. Jpn. 84, 024704 (2015).

27) A. Yamanaka and S. Okada, Carbon 96, 351 (2016)

28) P. Hohenberg and W. Kohn, Phys. Rev. 136, B864 (1964).

29) W. Kohn and L. J. Sham, Phys. Rev. 140, A1144 (1965).

30) Y. Morikawa, K. Iwata, and K. Terakura, Appl. Surf. Sci. 169-170, 11 (2001).

31) J. P. Perdew, K. Burke, and M. Ernzerhof, Phys. Rev. Lett. 77, 3865 (1997).

32) J. P. Perdew, K. Burke, and M. Ernzerhof, Phys. Rev. Lett. 78, 1396 (1997).

33) D. Vanderbilt, Phys. Rev. B 41, 7892 (1990).

34) M. Maruyama, N. T. Cuong, and S. Okada, Carbon 109, 755 (2016).

35) M. Maruyama, N. T. Cuong, and S. Okada, J. Phys. Soc. Jpn. 84, 084706 (2015). 